\title{
Connaissances des travailleurs en radioprotection - Enquête au CHU Mongi Slim à La Marsa (Tunisie)
}

\author{
I. Marzouk Moussa ${ }^{\star}$ et H. Kamoun \\ Faculté de médecine de Tunis, Service de radiologie, CHU Mongi Slim, La Marsa, Sidi Daoued, Tunisie.
}

Reçu le 28 septembre 2015 - Accepté le 18 décembre 2015

\begin{abstract}
Résumé - Le but de notre travail était d'évaluer les connaissances en radioprotection du personnel hospitalier directement affecté aux travaux sous rayonnements ionisants (DATR). Nous avons réalisé une étude descriptive transversale, auprès de ce personnel dans les salles opératoires d'orthopédie, de cardiologie interventionnelle et du service de radiologie diagnostique et interventionnelle de l'hôpital Mongi Slim à la Marsa (Tunisie), en utilisant un questionnaire. Le score global des connaissances (SGC) a été calculé à partir du nombre de bonnes réponses aux questions $(n=20)$. Cinquante-quatre personnes ont participé à l'enquête, soit un taux de participation de $58 \%$. L'âge moyen était de 36 ans (VL : 25-63). Le SGC était de 11,8 (écart-type : 2,9) et environ 1 personne sur 2 avait un score moyen inférieur à 12. Le test de Kruskal-Wallis a montré que le niveau de connaissance variait significativement en fonction de la catégorie professionnelle, du service d'affectation et de l'ancienneté. Le score moyen au service de radiologie était meilleur qu'aux autres services. Le port du dosimètre n'était pas respecté par $70 \%$ (38 personnes) du personnel interrogé pour cause de non disponibilité. $83 \%$ (45 personnes) des personnes interrogées ont manifesté leur intérêt à suivre une formation en radioprotection.
\end{abstract}

\begin{abstract}
Staff knowledge of radiation protection - A Survey in the University Hospital of Mongi Slim La Marsa (Tunisia). The aim of our study was to assess the knowledge of radiation protection of hospital staff directly assigned to work with ionizing radiation (DATR). We conducted a cross-sectional descriptive study, with the DATR personnel in the orthopedic, interventional cardiology, and diagnostic and interventional radiology operating rooms of the Mongi Slim University hospital in La Marsa (Tunisia), using a self-administered questionnaire. The overall knowledge score (OKS) was calculated from the number of correct answers to the evaluation questions $(n=20)$. Fiftyfour people participated in the survey, with a response rate of 58\%. The average age was 36 years (LV: 25-63). The OKS was 11.8/20 (SD: 2.9), and about 50\% had an average score less than 12/20. The Kruskal-Wallis test showed that the level of knowledge varied significantly depending on the professional category, the assignment department and seniority. The average score in the radiology department was better than the other ones. Wearing a dosimeter was not complied with by $70 \%$ (38 persons) of the staff interviewed due to non-availability. $83 \%$ (45 persons) of respondents expressed interest in being trained in radiation protection.
\end{abstract}

Keywords: radiation protection / knowledge / data collection / radiology / Tunisia

\section{Introduction}

Selon le rapport 2008 du comité scientifique de l'organisation des nations unies (UNSCEAR) sur les conséquences des émissions radioactives, la dose efficace collective totale des examens de diagnostic médical a augmenté de $70 \%$ entre 1997 et 2007 (UNSCEAR, 2008). Le nombre des travailleurs directement affectés aux travaux sous rayonnements ionisants (DATR) a aussi augmenté d'un facteur 7 et la moyenne de la dose annuelle reçue par ces travailleurs a augmenté d'un facteur 1,7 de 1994 à 2002 selon le même rapport.

\footnotetext{
^ marzouk.ines@gmail.com
}

La Tunisie n'a pas échappé pas à cette tendance (Amri et al., 2007; République Tunisienne, 2013). En effet, ces 20 dernières années, le nombre d'examens irradiants diagnostiques et thérapeutiques a connu un véritable essor et plusieurs appareils plus irradiants tels que les scanners multidétecteurs ont été mis en place avec un recrutement d'un bon nombre de travailleurs DATR.

La radioprotection est rendue obligatoire en Tunisie conformément à la loi relative aux rayonnements ionisants (loi 81-51 du 18 juin 1981; République Tunisienne, 1986). Le Centre National de Radioprotection (CNRP), structure d'application de cette réglementation et disposant de moyens 
modestes, ne cesse depuis sa création de promouvoir les mesures et les méthodes destinées à la protection contre les dangers des rayonnements ionisants (RI).

La radioprotection des patients, des travailleurs et des membres du public repose sur les principes de justification des pratiques, d'optimisation des expositions et de limitation des doses reçues par les travailleurs (ICRP, 1990; 2007).

Aussi, la radioprotection des travailleurs rend obligatoire le respect des limites des doses de RI à ne pas dépasser chez les travailleurs conformément aux recommandations de la Commission Internationale de Protection Radiologique (CIPR) (ICRP, 2007). Afin de pouvoir répondre à ces obligations, les professionnels de la santé, médecins et paramédicaux, doivent être formés à la radioprotection.

La situation de la formation en radioprotection est préoccupante en Tunisie. Très peu d'études ont évalué les connaissances en radioprotection chez les travailleurs tunisiens. Les résultats d'une étude récente (Kamoun et al., 2015) reflètent un niveau faible chez les travailleurs DATR dans les salles opératoire d'orthopédie dans le grand Tunis (gouvernorats de l'Ariana, de Ben Arous, de la Manouba et de Tunis). Une étude portant sur l'état de la radioprotection du patient en médecine nucléaire (Ghezaiel et al., 2013) a relevé un certain nombre de manquements.

\section{Matériels et méthodes}

Une étude descriptive, transversale et exhaustive a été réalisée en mai 2015 au CHU Mongi Slim de La Marsa (Tunisie), auprès de tout le personnel DATR en radiologie, cardiologie interventionnelle et chirurgie orthopédique. La population cible comportait un effectif de 93 individus au moment de l'enquête.

Afin d'évaluer les connaissances du personnel en radioprotection, nous avons utilisé un questionnaire élaboré selon l'approche normative suggérée par l'Agence Internationale à l'Énergie Atomique (IAEA, 2005) et après une analyse des données de la littérature (Shiralkar et al., 2003 ; Ongolo-Zogo et al., 2013 ; Günalp et al., 2014; Yurt et al., 2014). Ce questionnaire a été remis à chaque personne consentante pour participer à notre étude $(n=54)$. Il comportait sept parties évaluant :

1. les caractéristiques socio-professionnelles : sexe, âge, grade, service d'affectation et ancienneté au poste exposant aux rayonnements ionisants (RI);

2. les connaissances concernant les caractéristiques des RI;

3. les connaissances des moyens de protection contre les RI;

4. les connaissances des moyens de surveillance de l'exposition aux RI;

5. les connaissances des effets sanitaires des RI;

6. la connaissance des moyens de radioprotection et les valeurs limites annuelles d'exposition à ne pas dépasser ;

7. le comportement à tenir en cas d'accident d'exposition aux RI.

Le score global des connaissances (SGC) a été comptabilisé à partir du nombre de bonnes réponses aux questions $(n=20)$.

Les scores ont été classés en deux niveaux. Pour des raisons statistiques nous avons groupé les scores classés bons (scores supérieurs à 16) et les scores classés moyens (scores compris entre 12 et 16) en un seul groupe : moyens à bons (scores supérieurs à 12). Le deuxième groupe et celui des mauvais scores (scores inférieurs à 12).

Pour l'analyse statistique, nous avons utilisé le logiciel SPSS (logiciel SPSS version 19.0 (IBM $\left.{ }^{\circledR}\right)$ ). Dans une première étape nous avons calculé des fréquences absolues et des fréquences relatives (pourcentages) pour les variables qualitatives. À l'aide du tableur Excel ${ }^{\complement}$ (Microsoft Office ${ }^{\circledR}$ ), nous avons calculé des moyennes, des médianes et des écarts-types et déterminé les valeurs extrêmes pour les variables quantitatives. Dans une deuxième étape, nous avons effectué une étude comparative entre les facteurs socioprofessionnels et les connaissances en radioprotection. Nous avons réalisé une étude analytique monovariée testant les variables une à une afin d'identifier les facteurs pouvant avoir un impact sur les connaissances des travailleurs.

Les comparaisons des moyennes sur des séries indépendantes ont été effectuées au moyen du test $\mathrm{t}$ de Student pour les séries indépendantes. Les comparaisons de pourcentage sur séries indépendantes ont été effectuées par le test de Chi-deux de Pearson et en cas de non validité de ce test, par le test de Kruskal-Wallis. Une différence entre deux groupes a été considérée statiquement significative si $p$ est inférieur à 0,05 .

\section{Résultats}

\subsection{Les caractéristiques socioprofessionnelles de notre population d'étude}

Uniquement 54 personnes sur 93 ont répondu au questionnaire. L'âge moyen était de 36 ans (VL : 25 à 63) avec une médiane de 34 ans. La répartition selon le sexe a mis en évidence 33 femmes et 21 hommes soit un sexe ratio de 0,6. La répartition selon les services a mis en évidence 32 travailleurs du service de radiologie, 8 de la cardiologie et 14 de la salle opératoire d'orthopédie (Tableau 1). La répartition selon le grade a mis en évidence 7 médecins diplômés, 13 médecins en formation, 27 techniciens supérieurs et 7 infirmiers. La majorité du personnel enquêté était des techniciens supérieurs $n=27$ (50\%). Quatre-vingt pourcent des enquêtés de l'étude appartenaient à la catégorie A. La répartition selon l'ancienneté professionnelle au poste exposant aux RI a mis en évidence 24 travailleurs ayant moins de 5 ans d'expérience, 15 entre 5 et 10 ans et 15 plus de 10 ans.

\subsection{Le taux de participation selon les services}

Le taux de participation a été calculé selon le rapport entre le nombre de personnes du service ayant participé et l'effectif du personnel dans le service. Le taux de participation était le plus élevé (62\%) pour le service de radiologie.

\subsection{Répartition de la population étudiée selon le score global des connaissances (SGC)}

Dans la population étudiée, le SGC était de 11,8 avec des valeurs limites de 1 à 18 . Vingt-deux personnes (40,7\%) 
Tableau 1. Répartition de la population enquêtée selon les caractéristiques socioprofessionnelles et la moyenne des scores.

\begin{tabular}{|c|c|c|c|c|}
\hline & & $n$ & $\%$ & Moyenne des score /20 \\
\hline \multicolumn{5}{|l|}{ Sexe } \\
\hline & Féminin & 33 & $61 \%$ & 11,4 \\
\hline & Masculin & 21 & $39 \%$ & 12,4 \\
\hline \multicolumn{5}{|c|}{ Grade professionnel } \\
\hline & Médecin diplômé (professeur / assistant) & 7 & $3 \%$ & 13,3 \\
\hline & Médecin en formation & 13 & $24 \%$ & 12,7 \\
\hline & Technicien supérieur & 27 & $50 \%$ & 10,9 \\
\hline & Infirmier & 7 & $13 \%$ & 11,4 \\
\hline \multicolumn{5}{|c|}{ Expérience cumulée en RI } \\
\hline & $<$ à 5 ans & 24 & $44 \%$ & 11,4 \\
\hline & 5 à 10 ans & 15 & $28 \%$ & 11,5 \\
\hline & $>$ à 10 ans & 15 & $28 \%$ & 12,7 \\
\hline \multicolumn{5}{|c|}{ Service } \\
\hline & Radiologie & 32 & $59 \%$ & 12,7 \\
\hline & Cardiologie interventionnelle & 8 & $15 \%$ & 10,6 \\
\hline & Salle opératoire d'orthopédie & 14 & $26 \%$ & 10,4 \\
\hline
\end{tabular}

avaient un score moyen à bon, tandis que $32(59 \%)$ avaient un mauvais score.

\subsection{Répartition du SGC selon les caractéristiques socio-professionnelles (Tableau 1)}

La moyenne des scores la plus élevée (13,3 - VL : 11 à 16) a été observée chez les médecins diplômés suivis par les médecins en formation (12,7 - VL : 10 à 18). La moyenne la plus basse a été observée chez les techniciens supérieurs $(10,9-$ VL : 1 à 15$)$ suivis par les infirmiers (11,4 - VL : 9 à 14). Le personnel du service de radiologie a obtenu une moyenne de SGC plus élevée (12,6 - VL de 8 à 18) comparativement aux deux autres services. La moyenne des SGC chez le personnel médical incluant les médecins diplômés (assistants et professeurs) et non diplômés (résidents) a été supérieure (13VL de 10 à 18) à celle du personnel paramédical $(11,2-$ VL : 1 à 15). Dans le sous-groupe des médecins, la moyenne des SGC des assistants a été la plus élevée (13,3 - VL de 11 à 16), mais la différence n'est pas statistiquement significative. Les travailleurs enquêtés ayant cumulé plus de 10 ans en zones DATR avaient un SGC significativement supérieur $(12,7)$ aux moins anciens $(p=0,004)$.

\subsection{Analyse détaillée des différents niveaux de connaissance}

\subsubsection{Connaissances concernant les caractéristiques des rayons $X$}

Cinq, soit $9 \%$ des participants ne connaissaient pas le sigle relatif aux RI. Quinze, soit $28 \%$ seulement des participants connaissaient la dose limite annuelle totale pour les travailleurs fixée par la CIPR à $20 \mathrm{mSv} \mathrm{an}^{-1}$ (ICRP, 2007).

\subsubsection{Connaissances concernant les moyens de protection contre les rayons $\mathrm{X}$}

Trente-neuf, soit $72 \%$ des enquêtés ont surestimé la distance minimale séparant un individu de l'amplificateur de brillance à partir de laquelle la dose reçue devient trop faible pour être détectée par le dosimètre (2 mètres) (Mehlman et DiPasquale, 1997). Au moment de l'enquête, les signalisations lumineuses d'émission de RI étaient présentes à l'entrée de toutes les salles dans le service de radiologie, absentes à l'entrée de la salle de bloc d'orthopédie et non fonctionnelles à l'entrée de la salle de cardiologie interventionnelle. Trentedeux pourcent des personnes enquêtées avaient noté leur présence ou leur état de fonction.

\subsubsection{Connaissances concernant les moyens de surveillance de l'exposition aux rayons $X$}

Les dosimètres étaient portés par seulement 16 personnes soit $29 \%$ du personnel enquêté. Pour ceux qui ont déclaré ne pas porter le dosimètre passif thermoluminescent, la non disponibilité de ce dernier était la cause pour 29 d'entre eux soit $54 \%$ des travailleurs enquêtés. Les 9 restants ou $17 \%$ ont déclaré ne pas porter intentionnellement le dosimètre car qualifié d'inutile ou défaillant. Il est à noter que l'institution n'est pas approvisionnée en dosimètres opérationnels.

\subsubsection{Connaissances concernant les effets des rayons $X$}

Quarante-et-un, soit $76 \%$ des travailleurs enquêtés connaissent les effets des RI sur la santé mais en ce qui concerne les effets spécifiques aux faibles doses, 26 soit $48 \%$ des enquêtés ont déclaré ne pas en connaitre les risques. Le cancer a été évoqué par $94 \%$ et la stérilité par $30 \%$ de ces personnes. Vingt-six, soit $48 \%$ des personnes enquêtées ne savaient pas qu'une personne atteinte de cataracte était inapte à travailler en zone DATR. Vingt-six et $46 \%$ des enquêtés respectivement, croient à tort que le diabète déséquilibré et la neuropathie sont un motif d'arrêt de travail en zone DATR. 


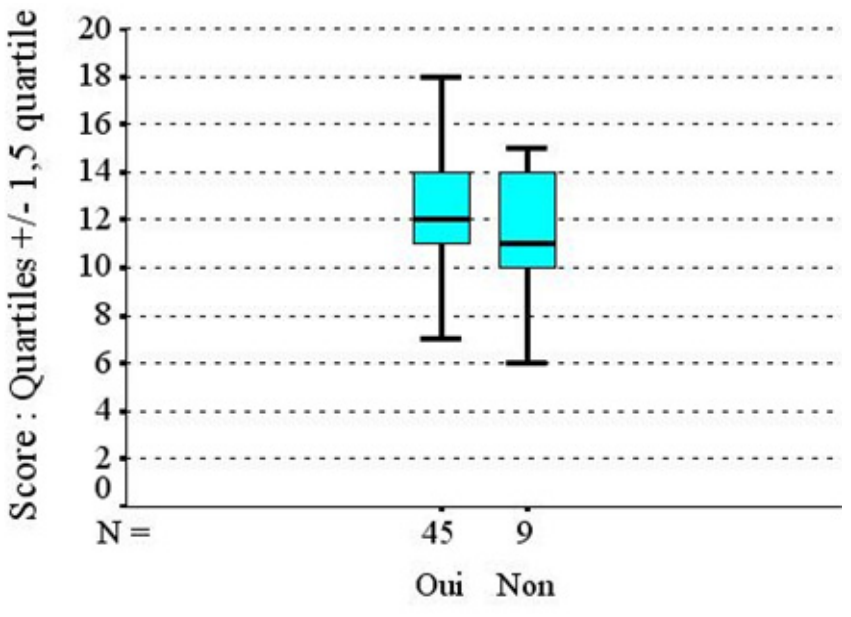

Intérêt pour une formation

Figure 1. Répartition des moyennes des scores selon la motivation pour suivre une formation.

\subsubsection{Connaissances concernant les normes de radioprotection}

Deux enquêtés seulement ont répondu correctement et de façon complète à la question portant sur les limites annuelles d'exposition aux RI spécifiques aux extrémités, au cristallin, au corps entier et au fœtus.

\subsection{Comportement en cas d'exposition accidentelle aux RI}

La conduite à tenir devant la survenue d'un accident d'exposition aux RI définie par la loi Tunisienne (République Tunisienne, 1981, 1986) a été correcte pour 38 personnes soit $70 \%$ des cas. Cependant, seulement $17 \%(n=9)$ connaissaient la personne compétente en radioprotection dans le service ou l'institution qu'il faudrait contacter dans ce cas.

Enfin, 45 sujets enquêtés soit $83 \%$ étaient motivés à suivre une formation en radioprotection. Les personnes non intéressées avaient un score moyen de 10,3 (plus bas que la moyenne générale) (Figure 1).

\section{Discussion}

Notre étude a visé un échantillon de la population du personnel tunisien DATR. Elle rend compte de la situation préoccupante du niveau de connaissance qui est loin d'être optimal notamment dans le rang du personnel paramédical.

La méthode de notre enquête comporte des avantages et des inconvénients.

L'inconvénient majeur est le taux de participation; il était de $58 \%$ dans notre enquête et il est jugé moyen comparé à d'autres études de même type (Ongolo-Zogo et al., 2013; Kamoun et al., 2015). Le taux de non répondants peut être expliqué par les congés et/ou la charge de travail importante qui rend le personnel moins disponible. Nous avons fixé le nombre de visites maximum à 3 au-delà desquelles la personne à enquêter est considérée non répondeuse.

Nous avons délivré un questionnaire anonyme. L'avantage de ce type de questionnaire est qu'il augmente le taux de participation. Cependant, l'inconvénient majeur est la surestimation du niveau de connaissances. En effet, le sujet enquêté peut consulter une référence sur internet ou un collègue. Nous avons essayé de pallier dans la mesure du possible ce biais en essayant d'écourter le temps laissé à l'enquêté pour remplir le questionnaire.

Notre étude n'a pas concerné les connaissances sur la radioprotection du patient à cause de l'hétérogénéité des sources de RI utilisées, des niveaux de références et des méthodes d'optimisation des doses délivrées selon le département. L'absence de services de médecine nucléaire ou de radiothérapie dans notre institution est un point faible pour notre étude. Ce travail, malgré ses limites a permis d'apprécier le niveau de connaissance dans une installation hospitalière de Tunis donc d'orienter les efforts à venir en termes de formation du personnel.

Ce travail pourrait être complété par une deuxième enquête visant une population cible plus large et plus représentative de l'ensemble des travailleurs hospitaliers DATR en Tunisie.

La dose maximale admissible (DMA) est, à l'origine, « la dose de RI qui ne doit causer aucune lésion corporelle appréciable chez une personne exposée, à aucun moment de sa vie » (Cordoliani, 2014). Les connaissances des normes dosimétriques étaient quasi nulles dans notre enquête aussi bien chez les médecins que chez le personnel paramédical.

Concernant les effets à long terme des faibles doses sur les travailleurs en milieu médical, une analyse de huit études de cohortes de radiologues et de techniciens en radiologie dans divers pays, comprenant 270000 travailleurs sous rayonnements surveillés (Yoshinaga et al., 2004), a conclu qu'il y avait un risque significatif de cancers parmi les premiers travailleurs avant 1950, mais aucune preuve de risque parmi les nouveaux travailleurs. Dans notre étude, la possibilité de survenue d'effets stochastiques aux faibles doses était méconnue par $48 \%$ des enquêtés sans doute à cause d'un manque de sensibilisation et de formation.

La défaillance de la surveillance dosimétrique individuelle a également été observée dans une étude évaluant les connaissances en radioprotection des travailleurs au bloc d'orthopédie du Grand Tunis (Kamoun et al., 2015). La panne du lecteur des dosimètres thermoluminescents au CNRP et la difficulté subie par l'établissement pour l'approvisionnement en nombre suffisant de dosimètres opérationnels sont les causes principales de la défaillance actuelle de la surveillance dosimétrique en Tunisie.

Il n'existe pas de questionnaire en langue française standardisé pour l'évaluation de la connaissance du personnel travaillant en RI, toutefois des études similaires faites dans des centres hospitaliers universitaires ont trouvé un niveau de connaissances insuffisant en Turquie (Yurt et al., 2014), en Éthiopie (Zewdneh et al., 2012), en Tunisie (Kamoun et al., 2015) ainsi qu' au Cameroun (Ongolo-Zogo et al., 2013), et un bon niveau au Nigeria (Adejumo et al., 2012).

Selon le rapport de la CIPR (ICRP, 2012), la dose maximale annuelle cumulée au cristallin a été abaissée de 
$150 \mathrm{mSv}^{-1}$ à une moyenne de $20 \mathrm{mSv} \mathrm{an}^{-1}$ sur 5 ans sans dépasser $50 \mathrm{mSv}$ en une seule année. Le risque relatif de survenue d'une cataracte dans la population de cardiologues interventionnels par rapport au reste de la population varie de 2,6 à 3,3 selon les séries (Ciraj-Bjelac et al., 2012). Ceci nous incite à souligner dans nos résultats la méconnaissance de cet effet par $48 \%$ des enquêtés. Une surveillance dosimétrique spécifique du cristallin est proposée actuellement pour le personnel DATR notamment en radiologie interventionnelle (IRSN, 2013). Les nouvelles lunettes plombées apportent une atténuation d'au moins $90 \%$ du rayonnement incident et présentent un bon compromis poids-efficacité (Koukorava et al., 2011).

Nous avons constaté l'influence significative du grade professionnel sur le niveau de connaissances entre le personnel médical et paramédical. Cela suggère l'importance et la pertinence de développer les enseignements sur la radioprotection lors de la formation initiale des techniciens supérieurs de santé et des infirmiers. Dans la population des médecins, la différence a été significative entre les radiologues et les non radiologues. La formation initiale des médecins orthopédistes et cardiologues interventionnels devrait être obligatoire dans le cadre du collège de la spécialité. La formation continue doit également être renforcée pour cette catégorie de médecins. Actuellement, la méthode pédagogique la plus adaptée à la réactualisation des connaissances semble être l'Évaluation des Pratiques Professionnelles (EPP). En France, l'EPP est rendue obligatoire pour les médecins par la loi du 13 août 2004 (République Française, 2004) tandis que l'enseignement de cette discipline pour les radiologues tunisiens a été laissé au grès des médecins sans évaluation ni éventuelle sanction.

Les recommandations de la CIPR (ICRP, 2013) ont mentionné l'importance de l'enseignement de la radioprotection dans le cadre de la formation professionnelle initiale ou continue. Elle constitue en effet un pilier essentiel en termes d'assurance de la sécurité des travaux réalisés.

L'expression par $83 \%$ des travailleurs enquêtés d'un besoin en formation continue en matière de radioprotection reflète la volonté de changer la situation. Pour garantir la réussite de la formation en radioprotection, nous allons prendre en considération les facteurs influençant l'adhésion des travailleurs aux normes de radioprotection qui sont de différents ordres :

- individuel : l'attitude vis-à-vis du risque, la norme sociale perçue, le contrôle comportemental et l'intention;

- organisationnel : la formation, la sensibilisation, les infrastructures et les moyens de protection, la surveillance du personnel et la sécurité au travail (Rehel, 2010);

- contextuel : les lois et les normes mises en œuvre pour encadrer cette formation.

Dans l'institution visée par notre enquête, le facteur organisationnel devrait être davantage renforcé avec un double objectif : sensibiliser et former les travailleurs.

Le constat de notre étude devrait interpeller les autorités hospitalières et de santé publique ainsi que les sociétés savantes sur la nécessité urgente d'exiger une formation agréée qualifiante en radioprotection non seulement vis-à-vis du travailleur lui-même mais également du patient et de la population. Une remise à niveau des professionnels est nécessaire en prenant comme cadre la directive EURATOM (Conseil de
l'Union Européenne, 1997). Le cadre règlementaire tunisien de la radioprotection n'a pas changé depuis 1986.

\section{Actions correctives}

Pour corriger les insuffisances constatées dans les connaissances des travailleurs en matière de radioprotection, nous avons planifié en coordination avec le CNRP deux sessions de formation au sein de l'institution visant deux groupes différents; les médecins et les paramédicaux. Les objectifs de ces sessions vont différer selon les résultats de chaque groupe. En effet, pour les médecins les points cruciaux à souligner porteront sur les grandeurs dosimétriques, les valeurs des limites annuelles des doses cumulées ainsi que sur les normes de radioprotection. Pour les paramédicaux, il sera nécessaire de rappeler les caractéristiques des RI, les normes de radioprotection ainsi que les moyens de surveillance. Avec la complicité de la personne compétente en radioprotection de l'institution, il sera utile d'expliquer le rôle du CNRP et la législation tunisienne en matière de radioprotection lors de ces sessions.

Des affiches de sensibilisations à la radioprotection ont également été conçues pour chaque service dans les zones surveillées.

Enfin, une deuxième enquête sera réalisée après la formation pour évaluer son impact sur le personnel ayant suivi cet enseignement.

\section{Conclusions}

Cette étude montre que le niveau de connaissance en matière de radioprotection du personnel DATR dans le CHU Mongi Slim à La Marsa est globalement bas. Notre étude est d'abord quantitative, permettant d'évaluer le niveau de connaissances et de préciser les lacunes. La concrétisation pratique se fera par la sensibilisation et l'organisation de sessions de formation en radioprotection pour le personnel concerné. Il sera alors primordial d'impliquer tous les intervenants en radioprotection et d'évaluer la pertinence de cette formation avec une $2^{\mathrm{e}}$ enquête.

\section{Références}

Adejumo S.B., Irurhe N.K., Olowoyeye O.A., Ibitoye A.Z., Eze C.U., Omiyi O.D. (2012) Evaluation of Compliance to Radiation Safety Standard Amongst Radiographers in Radiodiagnostic Centres in South West, Nigeria, WJMS 7 (3), 194-196.

Amri C., Chaari N., Bedoui M.H., Henchi M.A., Abdallah B., Ben Hamda K., Gamra H., Akrout M., Khalfallah T. (2007) Évaluation de l'irradiation ionisante des opérateurs en cardiologie interventionnelle, Feuillets de Radiologie 47 (1), 3-8.

Ciraj-Bjelac O., Rehani M., Minamoto A., Sim K.H., Liew H.B., Vano E. (2012) RadiationInduced Eye Lens Changes and Risk for Cataract in Interventional Cardiology, Cardiology 123 (3), 168171.

Conseil de l'Union Européenne (1997) Directive 97/43/EURATOM du 30 juin 1997 relative à la protection sanitaire des personnes contre les dangers des rayonnements ionisants lors d'expositions à des fins médicales. 
Cordoliani (2014) Fondements de la radioprotection. Dans : Radioprotection en milieu médical - Principes et mise en pratique (Y.S. Cordoliani, H. Foehrenbach Eds.), pp. 1-8. Elsevier Masson, Issy-les-Moulineaux.

Ghezaiel M.J., Slim I., Mayna H., El Bez I., Mhiri A., Ben Slimène M.F. (2013) La radioprotection des patients en médecine nucléaire : état des lieux en Tunisie, Médecine Nucléaire 37, 586590.

Günalp M., Gülünay B., Polat O., Demirkan A., Gürler S., Akkaş M., Aksu N.M. (2014) Ionising Radiation Awareness among Resident Doctors, Interns, and Radiographers in a University Hospital Emergency Department, Radiol. Med. 119 (6), 440-447.

IAEA (2005) Building Competence in Radiation Protection and the Safe Use of Radiation Sources jointly sponsored by IAEA, ILO, PAHO, WHO. Safety standards series No. RS-G-1.4. IAEA, Vienna.

ICRP Publication 60 (1990) Recommendations of the International Commission on Radiological Protection, Ann. ICRP 21.

ICRP Publication 103 (2007) The 2007 Recommendations of the International Commission on Radiological Protection, Ann. ICRP 37.

ICRP Publication 118 (2012) ICRP Statement on Tissue Reactions and Early and Late Effects of Radiation in Normal Tissues and Organs - Threshold Doses for Tissue Reactions in a Radiation Protection Context, Ann. ICRP 41.

ICRP Publication 120 (2013) Radiological protection in cardiology, Ann. ICRP 42.

IRSN (2013) Recommandations sur les bonnes pratiques en matière de radioprotection des travailleurs dans la perspective de l'abaissement de la limite réglementaire de dose équivalente pour le cristallin. Rapport PRP-HOM/2013-0001.

Kamoun H.D., Abbes K., Kamoun A., Attia N., Hammou A. (2015) Connaissances du personnel en radioprotection - étude multicentrique en chirurgie orthopédique sur le grand Tunis, Arch. Mal. Prof. 76 (3), 269-278.

Koukorava, C., Carinou E., Ferrari P., Krim S., Struelens L. (2011) Study of the Parameters Affecting Operator Doses in Interventional Radiology Using Monte Carlo Simulations, Radiat. Meas. 46 (11), 1216-1222.

Mehlman Ch.T., DiPasquale Th.G. (1997) Radiation Exposure to the Orthopaedic Surgical Team Fluoroscopy : How Far Away Is Far Enough ?, J. Orthop. Trauma. 11 (6), 392-398.
Ongolo-Zogo P., Nguehouo M.B., Yomi J., Nko'o Amven S. (2013) Connaissances en matière de radioprotection : enquête auprès des personnels des services hôspitaliers de radiodiagnostic, radiothérapie et médecine nucléaire à Yaoundé, Cameroun, Radioprotection 48 (1), 39-49.

Rehel J.L. (2010) Obligations du radiologue pour la radioprotection des travailleurs, J. Radiol. 91 (11), 1212-1219.

République Française (2004) Article L.4133-1-1 du Code de la Santé Publique, http://www.legifrance.gouv.fr/

République Tunisienne (1981) Loi $\mathrm{N}^{\circ}$. 81-51 concernant la protection contre les dangers des sources de rayonnements ionisants, Journal Officiel de la République Tunisienne : 18 juin.

République Tunisienne (1986) Article No. 24 décret No. 86.433 concernant la protection contre les rayonnements ionisants, Journal Officiel de la République Tunisienne : 11 avril.

République Tunisienne (2013). Carte sanitaire 2011, Ministère de la santé Publique, Direction des Études et de la Planification.

Shiralkar S., Rennie A., Snow M., Galland R.B., Lewis M.H., GowerThomas K. (2003) Doctors' knowledge of radiation exposure : questionnaire study, BMJ 327 (7411), 371-372.

UNSCEAR (2008). United Nations Scientific Committee on the Effects of Atomic Radiation UNSCEAR 2008 Report to the General Assembly, Volume I : Report to the General Assembly with Scientific Annexes A : medical radiation exposures and B : Exposures of the public and workers from various sources of radiation. New York, United Nations.

Yoshinaga S., Mabu K., Sigurdson A.J., Morin Doody M., Ron E. (2004) Cancer Risks among Radiologists and Radiologic Technologists : Review of Epidemiologic Studies, Radiology 233 (2), 313-321.

Yurt A., Berrin Ç. Türkan G. (2014) Evaluation of Awareness on Radiation Protection and Knowledge About Radiological Examinations in Healthcare Professionals Who Use Ionized Radiation at Work, Mol. Imaging Radionucl. Ther. 22 (2), 48-53.

Zewdneh D., Dellie S.T., Ayele T. (2012) A Study of Knowledge \& Awareness of Medical Doctors towards Radiation Exposure Risk at Tikur Anbessa Specialized Referral and Teaching Hospital, Addis Ababa, Ethiopia, J. Pharma. Bio. Sci. 2, 1-5. 